\title{
Modeling Spacetime as a Branched Covering Space over 2-Knots
}

\author{
Christopher Duston \\ Merrimack College, Department of Physics \\ dustonc@merrimack.edu
}

\begin{abstract}
In this paper we review a proposal to represent the geometric degrees of freedom of the gravitational field as a branched covering space, and introduce a new application of this in which the branch loci are 1- or 2-knots. This allows one to construct arbitrary smooth, closed 3- and 4-manifolds with enough geometric and topological information to write down a partition function and calculate statistical quantities in the thermodynamic limit. Further, we find clear evidence for a dimensional reduction of the spacetime geometry from four to two. As an example, we choose a family of smooth 4-manifolds presented in this way, and calculate the entropy of the system.
\end{abstract}

Keywords - general relativity, geometry, topology, branched covering space, exotic smooth structure, knot theory

\section{Introduction}

Differential geometry and topology are fundamentally important to the analysis of spacetime models. Not just because the physical theory which describes spacetime is built upon them, but they are also naturally associated with the physical concept of space. Like so many close connections in theoretical physics and mathematics, interesting features and results from one inform the explorations of the other. A specific example of this is that of the classification problem of smooth 4-manifolds, in which the key issue is exotic smooth structure. Attempts to understand and model exotic smooth structure have lead to new threads of exploration, such as the paradigm of using branched covering spaces and partition functions to study semiclassical gravity that will be utilized in this paper.

What we present here is a particular reparameterization of the gravitational field. In the traditional approach to gravitational theory, the field variable is the metric, which lives on a smooth 4-manifold. Briefly, a 4-manifold is a mathematical structure which provides a background upon which to precisely define the usual operations of calculus, but on a curved surface. In order for the early relativists to postulate that "spacetime is curved", this mathematical structure was needed so that one could define what it would mean for a test mass to move on such a curved surface. To build a manifold, you begin with points, collect these points together into sets, define coordinate systems on those sets, and then define transition functions on the sets which allows motion between them. The manifold becomes smooth (or topological, or piecewise-linear) when the transition functions are required to be infinitely differentiable (or continuous, or piecewise-continuous).

Once this structure is in place, the metric can be defined on the tangent space at points of the spacetime. Of course, the nature of the Einstein equations is such that one does not build the manifold first; the metric and the matter influence each other and are inexorably linked. This background independence marks general relativity as unique from all other field theories. If we find a consistent metric and matter configuration, we are implicitly assuming that a manifold could be constructed which furnishes such a metric. Solving the Einstein equations then amounts to solving a set of second-order coupled partial differential equations. Not only is this quite a difficult task except for only the very simplest of manifolds and matter distributions, but the precise nature of the spacetime manifold is generally hidden. Topological characteristics are difficult to detect, and physical results are necessary restricted to local regions of the spacetime, where the metric has been determined.

The approach followed in this paper will allow us to recover some of this lost information. The backbone of this construction is a branched covering space, and the geometric and topological information will be encoded in 2dimensional submanifolds of an auxiliary 4-sphere. In essence, we will describe how to construct any closed, smooth model for a 4-dimensional spacetime as number of 4-spheres sewn together along surfaces.

By doing this, some information will be retained about the spacetime model that is actually being used. Since the topological structure is recorded, it will be known from the outset. In addition, since this can be done generically, calculations which require a "sum over states" can now be formally complete. The only information which is lost in 


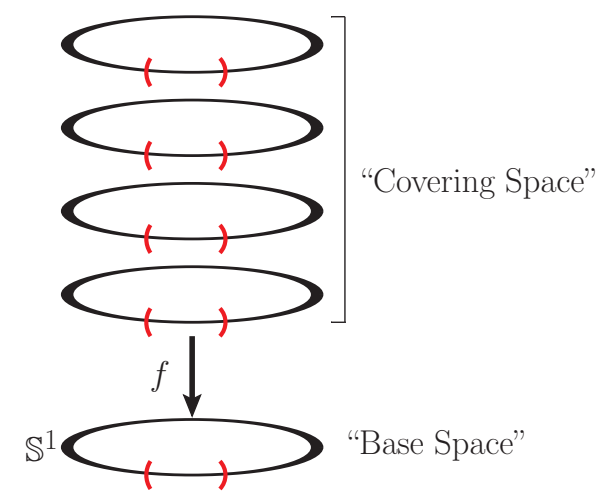

Figure 1: A 4-fold covering space of the 1-sphere, illustrating that the preimage of every open set in the base is an identical copy of the open set in the cover.

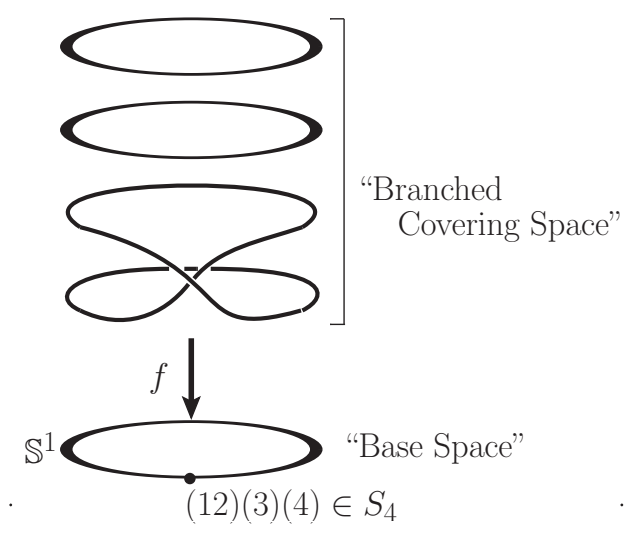

Figure 2: A 4-fold branched covering space of the 1sphere. This is a regular covering space everywhere except for a single point, over which there are only three preimages.

this approach is the physical intuition for the associated spacetime, but there is no fundamental reason why this is true, only that our originates from the standard parameterization. Since any 4-manifold can be presented in this way, one can alternate between the traditional representation, in which the topology is obscured but the field equations solved, and this alternative representation using a branched covering space.

The rest of this work will be presented as follows. We will begin by introducing branched covering spaces in 3and 4-dimensions ( $\$ 2$ ), and then discuss using 1- and 2-knots as branch loci (\$3). We will then relate the singularities in the base to those in the cover (\$4), and discuss how we will relate these singularities to the knot type of the locus (\$5). Then we will present our two primary results, dimensional reduction, demonstrated with a partition function approach (\$6), and a specific example of a 3-fold branched cover over a trefoil (77). We will conclude in 8 with a summary and outlook for the future.

\section{Branched Covering Spaces as Representations of 3- and 4-Manifolds}

The possibility of presenting a spacetime model as a branched covering space has been explored previously by the author and others [26, 12, 13, 14, 15, 16. In this approach, the base space of a covering space serves as something of a prototype, copies of which are sewn together to create the spacetime model. The sewing is defined by the covering space, which can be described using a representation of a permutation group corresponding to deck transformations. These approaches are generic enough to enable the construction of any closed manifold in 3- or 4-dimensions, which makes them particularly convenient for studying large classes of spacetime models. Depending on the context, statements like "all the closed spacetimes of dimension $d$ " with some particular parameters can be made precise.

We begin by carefully defining the mathematical constructions we will be using.

Definition 1. A covering map $p: M \rightarrow B$ is a continuous, surjective map between topological manifolds $M$ and $B$ such that for every open set $U \subset B$ the inverse image $p^{-1}(U)$ can be written as the union of disjoint open sets $V_{i} \subset M$ such that $\left.p\right|_{V_{i}}$ is a homeomorphism of $V_{i}$ onto $U . B$ is called the base space and $M$ is called the covering space. The number of inverse images $m$ in $p^{-1}(U)$ is called the order of the covering.

An example of a 4-fold covering space of the 1-sphere is shown in figure 1. The copies of the base in the cover are referred to the sheets, and when these sheets "collide" we get a branched covering space:

Definition 2. A map $p: M \rightarrow B$ is a branched covering map if there is a subset $L \subset B$ such that the restriction of $p$ to $M-p^{-1}(L)$ is a covering map. The set $L$ is called the branch locus, the preimage of the branch locus is the ramification locus, and $M$ is a branched covering space.

This is illustrated in figure 2 It should be noted that although the base is the same, the cover is topologically distinct, being four disjoint copies of the base in the first case as compared to three disjoint copies for the branched. It should also be emphasized that the branched cover spaces are smooth, non-singular 4-manifolds. The singular structure is completely contained in the covering map, which is singular when evaluated on the ramification locus. 


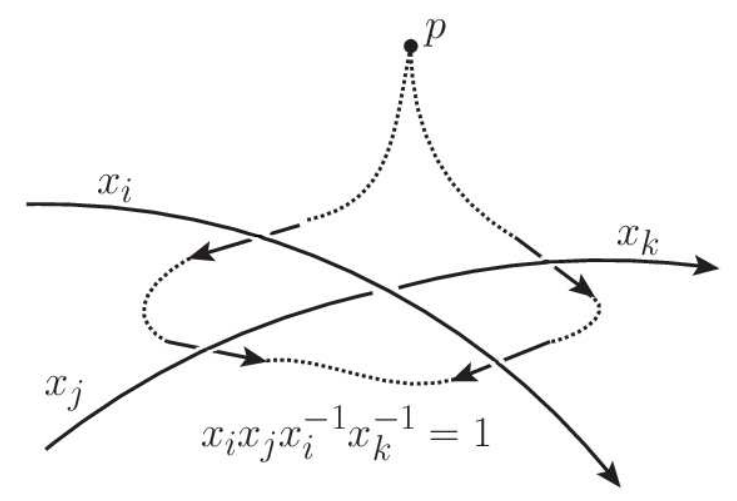

Figure 3: The definition of a vertex relation for the presentation of a knot group.

Clearly, the exact nature of the branched covering space depends on the base, the branch locus, and how the sheets "collide" over the locus. A convenient (and complete) way to specify this is an element of the permutation group attached to the ramification locus (see figure 21). This permutation group provides topological information about how the multiple copies of the base are sewn together to form the cover. Technical details of this construction can be found in the next section.

It turns out that the description of closed manifolds in this manner is complete, at least for dimensions of physical interest. It is a classic result of Alexander [1, 8] that this can be done in any dimension $p$ over $p$-spheres, branched along $(p-2)$ complexes. More recent work has demonstrated that one only needs 3 -fold covers in three dimensions [24, 28, 30] to represent any smooth 3-manifold in this way. In dimension four the situation is similar, but depends on the singular character of the branch locus:

Theorem 1 (Montesinos-Piergallini). [29] Any smooth, oriented, closed 4-manifold can be represented as a simple 4-fold covering of the 4-sphere branched over an immersed piecewise linear (PL) surface.

This result can actually be strengthened by restricting to locally flat PL surfaces at the cost of adding a 5th sheet 25], but since our branched loci will actually be non-locally flat, we will only be using the theorem as presented above.

\section{1- and 2-dimensional Knots as the Branch Locus}

Clearly, utilizing such representations of closed manifolds as spacetime models will only be productive if there is a straightforward way of keeping track of both the branch locus and the permutation labels presented in $\$ 2$ Fortunately, such techniques are well-known in knot theory, which is concerned with the classical problem of the equivalences of circles $\mathcal{K} \sim \mathbb{S}^{1}$ embedded in 3-spheres. There are a number of excellent introductions to this field [10, 19, 31, 30], and we will briefly review the key material here.

Definition 3. A p-knot $\mathcal{K}_{p} \simeq \mathbb{S}^{p}$ is an embedding $\mathbb{S}^{p} \rightarrow \mathbb{S}^{p+2}$. A link is a disjoint union of knots $\mathcal{K}_{p}^{(1)} \cup \mathcal{K}_{p}^{(2)} \cup \ldots \cup \mathcal{K}_{p}^{(n)}$. Two knots or links $\mathcal{K}_{p}, \mathcal{K}_{p}^{\prime}$ are equivalent if there is a homeomorphism $h: \mathbb{S}^{p+2} \rightarrow \mathbb{S}^{p+2}$ such that $h\left(\mathcal{K}_{p}^{\prime}\right)=\mathcal{K}_{p}$.

In this paper, we will only be concerned with the classical 1-knots and 2-knots. The equivalence of knots is easiest to (partially) determine by looking at the fundamental group of their complement, $G=\pi_{1}\left(\mathbb{S}^{p+2} \backslash \mathcal{K}_{p}\right)$, which is called the knot group (it is common to refer to both the knot and the image of the knot as $\mathcal{K}$, and we will not break this tradition). Intuitively, the fundamental group is the set of non-contractible paths under the operation of composition, and removing the knot from the sphere produces particular paths which are non-contractible. To determine a presentation of the knot group, a generator $x_{i}$ is assigned to each arc $i$ in the knot, and a set of relations $r_{i}$ for each vertex are defined, which correspond to paths traveling around that vertex. For example, see figure 3. These generators and relations form the group of a particular knot,

$$
G=\pi_{1}\left(\mathbb{S}^{p+2} \backslash \mathcal{K}_{p}\right)=\left(x_{1}, x_{2}, \ldots, x_{n} \mid r_{1}, r_{2}, \ldots, r_{m}\right)
$$

Once the knot group is found, there are a number of polynomial invariants which can be derived to determine if two knots are equivalent. We will not explore these in detail here, but examples are the Alexander polynomial, the 
Kauffman polynomial, and the Jones polynomial. What is important to know about these invariants is that they are incomplete - two knots with different sets of polynomial invariants are different, but two knots with equivalent polynomial invariants are not necessarily different.

In addition to the structure of the knot giving insight into the topology of the knot complement, it can also be used to determine the topology of covering spaces of the base sphere when branched over the knot. The essence is the following: as is clear in figure 2, we can think of the sheets being labeled with integers, and the "exchanging" of the sheets over the ramification locus to be a permutation label. Technically, to a given knot we assign a representation of the knot group $G$ into the symmetric group of order $n$, which matches the order of the cover:

$$
\rho: G \rightarrow \mathcal{S}_{n}
$$

Each arc of the knot has an associated element $g \in G$ in the following way: from any point in $B$ there is a nontrivial loop $f g f^{-1}$, where $f$ is a path from the base to the neighborhood of the arc, and $g$ is the element arbitrarily close to the arc. By associating each of these elements $g_{i}$ with a cycle in $\mathcal{S}_{n}$, we can describe what happens to paths which start on some sheet $j$, cross through the region of the preimage of the knot, and end up on sheet $k$. As discussed in [19, the set of admissible representations $\left\{\rho_{i}\right\}$ of a branched covering space can be indexed by the subgroups of $G$.

The fundamental group of the cover will be the pullback of the fundamental group in the base as long as the reconnections of the sheets over the branch points are taken account of. This can be done by applying the elements of the permutation group to the knot relations, as long as few technical details are dealt with. The full algorithm is detailed in 19, and for some examples of using this in practice see [14. The upshot is that given a knot and an assignment of permutation labels to each arc of the knot, we can reconstruct the branched covering space. In fact, in light of the presentation theorems discussed above, we can do this in full generality to construct any closed 3-manifold branched over 1-knots.

Of course, if we want to study spacetime models we want to be able to do the equivalent construction in dimension 4 , and it turns out there are several ways to do that using branched covering spaces. In previous work, this has been done by using algebraic curves of specified degree ([33, 13]), piecewise linear surfaces ([12, 14]), and the Weierstrass representation of surfaces [16] for the branch locus. Here we will represent the surfaces as 2-knots, so we want to extend the construction outlined above to that case.

An easy way to represent 2-knots (also called knotted spheres) is to consider the cross-sections of these surfaces. If the surface $\mathcal{K}_{2}$ is in general position, define the family of hyperplanes $H_{t}$ for $-\infty<t<\infty$ which intersects the knot for some values $t_{a}<t<t_{b}$. Restricted to each hyperplanes, the 2-knot with either be an image of a 1-knot or an isolated singular point. A singular point represents either the creation or destruction of a knot, or a reconnection of a knot vertex. Since each hyperplane contains a knot, one can determine the fundamental group of the complement of each level,

$$
\pi_{1}\left(H_{t} \backslash \mathcal{K}_{1}^{(t)}\right)
$$

and then determine the knot group $\pi_{1}\left(\mathbb{S}^{4} \backslash \mathcal{K}_{2}\right)$ of the van Kampen theorem, which essentially amounts to identifying reconnections. This entire process is presented in more detail in [19. Here we will just present an illustrative example, shown in figure 4

In the figure is shown a 2-knot with 1-knot (actually 1-link) cross-sections. In the hyperspace at $H_{t_{a}}$, there are two singularities, one of which is due to an unknot, and another which is due to a Hopf link (we will need to say a little bit more about the nature of this second singularity shortly). In this region, the fundamental group of knot complement in the hyperspace $H_{t}, t_{a}<t<t_{1}$ is given by

$$
\pi_{1}\left(H_{t} \backslash \mathcal{K}_{1}^{(t)}\right)=\left(a, b, c \mid a=b^{-1} a b\right) .
$$

When we reach $t_{1}$, two arcs of the link reconnect forming a singular point. Between $t<t_{1}$ and $t>t_{1}$, the generator $a$ is being identified with the generator $c$, and the fundamental group becomes $\left(a, b \mid a=b^{-1} a b\right)$. Upon crossing $t_{2}, a$ gets identified with $b$, and we get the final fundamental group of the 2-knot complement,

$$
\pi_{1}\left(\mathbb{S}^{4} \backslash \mathcal{K}_{2}\right)=(a) \simeq \mathbb{Z}
$$

It is easy to see that in our example, there is something different about the tips of the cone over the unknot as compared to the tip over the Hopf link. In the case of the unknot, every point on the cone has a region around it which looks like a disk (even the tip itself). This fails on the tip of the cone above the rings; as you take smaller and smaller neighborhoods around the tip, you still get ring cross-sections rather than disks. This is a case of the violation of local flatness:

Definition 4. For a submanifold $N^{n}$ of $M^{m}$, a point $p \in N$ is locally flat if it has closed neighborhood $U \in M$ such that $(U, U \cap N)$ is homeomorphic to the disks $\left(D^{m}, D^{n}\right)$. 


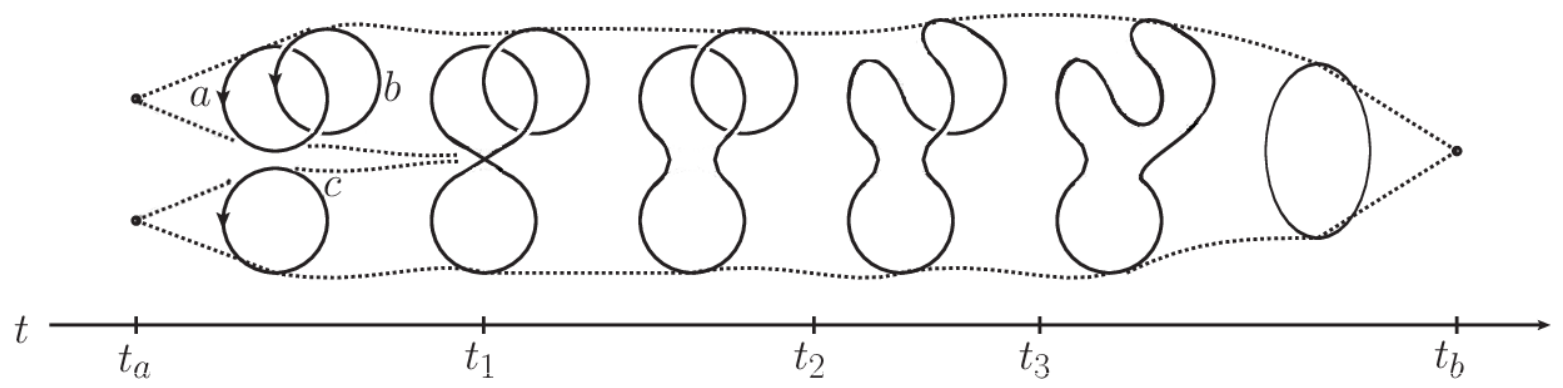

Figure 4

This definition is from [31. We can see that this condition is always satisfied for 1-knots; for every point of the knot, a neighborhood in $\mathbb{S}^{3}$ will be $U=D^{3}$, while $U \cap \mathcal{K}_{1}$ will be $D^{1}$. In the case of our example 2-knot in figure 4 , aside from the point at the tip of the cone over the ring, every point in $\mathcal{K}_{2}$ has a neighborhood homeomorphic to $D^{2}$. This point at the tip of the cone is a non-locally flat point, and will be a key feature of our later analysis.

Before moving on to the calculation of the curvature of these manifolds, we would like to point out that modeling spacetime as a branched covering space has been done previously in at least one notable case. It is natural to incorporate it into loop quantum gravity (LQG), because the spin networks used to track the geometry are already 1-dimensional complexes (graphs), and many of the techniques discussed here can be carried over. In [12, they show that for both the spin network and spin foam case, topological labels can be added (creating topspin networks and topspin foams) without modifying the graphs, which allows for the tracking of both geometry and topology in LQG. In 14, it is demonstrated that the algebra of LQG can be altered slightly to include this modification, and some example calculations are presented in [17].

\section{The Curvatures and Singularities of the Branched Cover}

Thus far we have shown how to construct 3- and 4-dimensional smooth manifolds as branched covers, with spheres for bases, branched over 1- and 2-knots. These manifolds can serve as spacetime models, and are generic. They are also conceptually simple - they are just copies of the base, sewn together in a way which is given by a knot, labeled with permutation indices. Geometrically they are spheres, with topology being recorded by a set of finite data packaged together in a convenient way.

Our concern here is to calculate the curvatures of the branched covers, so that we can use them to construct partition functions in the next section. Since these are just copies of 4 -spheres, we know what the curvature will be everywhere but near the non-locally flat points and the branch locus in the covers. We focus first on the non-locally flat points.

In the base sphere, these regions are cones, and can be assigned a metric of the form

$$
d s^{2}=d r^{2}+r^{2} d \phi^{2}+\left(\gamma_{i j}(\theta)+r^{2} h_{i j}(\theta)\right) d \theta^{i} d \theta^{j},
$$

where the $(r, \phi)$ coordinates are in the cone and $i, j, k, \ldots$ indices denote the coordinates $\theta^{1}$ and $\theta^{2}$, which are normal to the cone. Following [20], we will call the singular set $\Sigma$, which will be the $\left(\theta^{1}, \theta^{2}\right)$ plane that contains the singular point at $r=0$. The angular coordinate has a range $(0,2 \pi \alpha)$, and it is conventional to speak of the angular deficit $2 \pi(1-\alpha)$.

In [20], the scalar curvature ${ }^{(\alpha)} R$ of the singular manifold $M_{\alpha}$ is calculated by "rolling off" the singular surface to a regular manifold, calculating the curvature, and removing the regulator. The result is

$$
\int_{M_{\alpha}}{ }^{(\alpha)} R=4 \pi(1-\alpha) A_{\Sigma}+\int_{M_{\alpha} \backslash \Sigma} R
$$

where $R$ is now the scalar curvature of the manifold with the singular point removed, and $A_{\Sigma}$ is the area of the singular set, $\int \delta(\Sigma)$. What this means for us is that since the scalar curvature of the 4-sphere is constant, $\alpha$ and $\Sigma$ are our free parameters. For now we will move on to discussing the curvature near the branch locus, and we will discuss the angle deficit further in the next section.

Calling the neighborhood of the 2 -knot $N\left(\mathcal{K}_{2}\right)$, we can write the integral of the scalar curvature as

$$
\int_{\mathbb{S}^{4}} R=\int_{\mathbb{S}^{4} \backslash N\left(\mathcal{K}_{2}\right)} R+\int_{N\left(\mathcal{K}_{2}\right)} R .
$$


This expression will need to be pulled back to the covers through $p: M \rightarrow \mathbb{S}^{4}$. For a covering space of order $n$, the first term will simply give $n$ copies of the integral of the complement of a neighborhood of the knot. In the second term, on the singular surface $\Sigma$ there will be zero contribution to the integral because it is codimension 2 . It's also easy to see that just near the branch locus, integrals of constant curvature will simply be additive in the covering space (see 13] for example). If there are $m$ singularities (non-locally flat points) in the 2-knot there will be $r$ singular surfaces $\Sigma_{i}$ in the cover with angle deficits $\alpha_{i}$, for $m \leq r \leq r n$. This $r$ is not unknown, but could be determined with an analysis of the permutation labels. In the end, the integral of the action on the covers will be

$$
\int_{M} R=n \int_{\mathbb{S}^{4}} R+4 \pi \sum_{i}\left(1-\alpha_{i}\right) A_{\Sigma_{i}}
$$

\section{Singularity Type of the Knot and Angle Deficit}

The angular deficit, parameterized by $\alpha$, is a measure of "how conical" the spacetime around the cone is. There are several interesting features of geometric structures of this type. For instance, since any surface can be taken as a flat surface with singularities [38, we can associate those singularities with the conical ones and consider these surfaces to be flat. This is part of the origin of the equation (2) above.

In addition, conical metrics are used to describe one-dimensional topological structures in physical models of spacetime in the form of cosmic strings, with the tension related to the angular deficit [37. These are topological defects in the early universe which have not been observed but could contribute to a variety of phenomena, such as structure formation and inflation. The conical geometries have been used in previous studies of exotic smooth structure and topology of the gravitational field [13, 16, 18].

Here we will expand on the previous work by utilizing the 2-knot construction explicitly to determine the angular deficit of the comic string. The key feature which allows us to do this is the inclusion of the non-locally flat points discussed in the previous section. If the 2 -knot is locally flat, the metric is flat everywhere and there is no angle deficit. The presence of the non-locally flat point indicates the presence of an angle deficit, the size of which is directly related to the characteristics of the knot cross-section in the region around the conical point.

We will now restrict ourselves to a particular kind of knot, torus knots. This is a knot (or link) which lies on a torus in $\mathbb{S}^{3}$, wrapped around the latitude of the torus $p$ times and the longitude $q$ times. We call these $(p, q)$-torus knots. If $p$ and $q$ are not coprime, the knot forms completely disconnected parts and is actually a link. Torus knots can be described with a 1-parameter action on complex coordinates [27, 35]:

$$
t \cdot\left(z_{1}, z_{2}\right)=\left(t^{1 / p} z_{1}, t^{1 / q} z_{2}\right)
$$

for a $(p, q)$ torus knot, and the coordinates $\left(z_{1}, z_{2}\right)$ represent the latitude and longitude of the torus. This set of points is described by the polynomial

$$
f: \mathbb{S}^{3} \rightarrow \mathbb{C}, \quad f\left(z_{1}, z_{2}\right)=z_{1}^{p}+z_{2}^{q}
$$

when $f\left(z_{1}, z_{2}\right)=0$. The tangent spaces can be described with the derivative,

$$
D f: T \mathbb{S}^{3} \rightarrow T \mathbb{C}, \quad D f\left(z_{1}, z_{2}\right)=\left(p z_{1}^{p-1}, q z_{2}^{q-1}\right) .
$$

The inverse image of $f$ is a codimension 1 manifold everywhere except at $\left(z_{1}, z_{2}\right)=0$, since $D f=0$ vanishes there. So the origin is a singularity of the submanifold described by points which satisfy

$$
z_{1}^{p}+z_{2}^{q}=0 .
$$

We can develop a metric near these points in the following way. Begin with a flat metric on $\mathbb{R}^{4}$,

$$
d s^{2}=d x_{1}^{2}+d y_{1}^{2}+d x_{2}^{2}+d y_{2}^{2},
$$

and pick two sets of polar coordinates with periodicity $p$ and $q$ :

$$
\begin{array}{ll}
x_{1}=r_{1}^{p} \cos \left(p \phi_{1}\right), & y_{1}=r_{1}^{p} \sin \left(p \phi_{1}\right) \\
x_{2}=r_{2}^{q} \cos \left(q \phi_{2}\right), & y_{2}=r_{2}^{q} \sin \left(q \phi_{2}\right)
\end{array}
$$

Notice that this choice satisfies the defining equation (4) with $z_{1}=x_{1}+i y_{1}$ and $z_{2}=x_{2}+i y_{2}$. Pulling these back to the flat metric and enforcing the conditions $r_{1}^{p}=r_{2}^{q}, p \phi_{1}=q \phi_{2}$ and then rescaling $r=r_{1} \sqrt{2}$ and $\theta=(q / p) \phi_{2}$ we find

$$
d s^{2}=d r^{2}+\left(\frac{q}{p}\right)^{2} r^{2} d \theta^{2}
$$


This is a conical metric with $\alpha=q / p$.

We will pause here to emphasize what we have done. Conical metrics of the form (1) are well-studied, and used to represent topological defects with angle deficit $2 \pi(1-\alpha)$. $\alpha$ can be related to the string tension if the metric is modeling a string, but it is otherwise an unknown topological parameter of a 2-dimensional manifold. Here, we are using a fundamental feature of our particular spacetime model (torus knots which are cross-sections of the ramification locus) to characterize them, which gives us information about the topology of the spacetime. The topology is no longer a "free parameter", or something which cannot be kept track of, as is most common when studying solely local geometry.

One would naturally ask if this construction is specific to torus knots. Is it possible to generally write down a polynomial $f=\sum_{i j} z_{1}^{a_{i}} z_{2}^{a_{j}}=0$ which represents any knot? Recent work has shown that this is indeed the case [5], but the polynomials are complicated enough that we are unlikely to be able to find a simple set of coordinates like we could for the torus knots.

Finally, we must mention that there is a strict balance between the ability to make any surface flat with singularities and the angle deficit of those singularities. This comes from the Gauss-Bonnet theorem, which in the flat case is

$$
2 \pi \chi(\Sigma)+\sum_{i}\left(\theta_{i}-2 \pi\right)=0
$$

where $\chi(\Sigma)$ is the Euler characteristic of each surface containing non-locally flat points with angle deficits $\theta_{i}$ [20]. Since the angle deficits can be written $\theta_{i}=2 \pi\left(1-\alpha_{i}\right)$, this condition reduces to

$$
\chi(\Sigma)=\sum_{i} \alpha_{i}
$$

In other words, although we can always choose the knots to be locally flat, we cannot freely choose what the angle deficits will be; specifically, their sum has to be an integer. This could have already been a problem in our illustrative example (figure 4), but the Hopf link is a $(2,2)$-torus link, with $\alpha=1$. Therefore, as long as the other two singular points are unknots in a region arbitrarily close to them, this is a surface with $\chi(\Sigma)=1$, i.e. a projective plane.

\section{Partition Functions and Dimensional Reduction}

The formulation of the general theory of relativity in the language of thermodynamics, specifically with a partition function as a generating functional, is a useful approach when looking for new or interesting behaviors from an alternative representation of the gravitational field. Since it does not require a specific "belief structure" in regards to the quantum nature of the gravitational field (loops, strings, noncommutativity, etc.), it is a very flexible tool which allows one to study the transition region between classical and quantum gravity. It really only requires a belief that the transition between the classical and quantum gravitational field resembles that found in the other quantum fields, which is almost automatically true if one accepts the effective, Wilsonian viewpoint. Alternatively, since our experimental sophistication does not actually reach the quantum gravitational transition, these approaches can also be viewed as toy models for studying the abstract behavior of spacetime. For background into these approaches see [23, 22.

The partition function in our case is heuristically

$$
Z=\int_{\text {geometries }}[d g] e^{-\frac{1}{\hbar} S_{E H}[g]},
$$

where $g$ is the metric corresponding to a particular smooth manifold-metric pair $(M, g)$, and the action is the EinsteinHilbert one,

$$
S_{E H}=-\kappa \int_{M}(R-2 \Lambda) \sqrt{g} d^{4} x
$$

where $\kappa=c^{3} /(8 \pi G)$ for the case of Einstein-Hilbert gravity. This is the Euclidean action, which can be related to the Lorentzian one through a Wick rotation $t=-i \tau$.

A major problem with this definition is naturally the "integral over geometries", and is a partial motivation for our particular choice of branched covers and knots as representations of the spacetime model (e.g. the geometry). The traditional viewpoint is that, at least semiclassically, this integral is a sum over all gauge-inequivalent metrics. In the case of general relativity, the gauge symmetry is encoded by diffeomorphisms, so gauge-equivalence corresponds to metrics which are equivalent under coordinate changes (sometimes called passive diffeomorphisms [36], although we do not favor this categorization). However, the action is invariant under both passive and active diffeomorphisms (that is, all diffeomorphisms $f: M \rightarrow N$ between two smooth 4-manifolds), so the sum needs to be over all inequivalent pairs of complete geometries $(M, g)$. This complete geometry must specify both the topological and the smooth structure of the manifold. 
A complete determination of the diffeomorphism group Dif $f(M)$ for a particular manifold is difficult in general, and is an outstanding problem in dimension 4 . A particular feature of this unique situation is the presence of exotic smooth structure in dimension four. These are manifolds which are topologically equivalent but nondiffeomorphic, and represent gauge-inequivalent solutions to the Einstein field equations (for more details on exotic smooth structure see [34, 2]). The most dramatic example of this is that there are infinitely many exotic smooth structures on $\mathbb{R}^{4}[21$. The implications of this startling fact are far reaching, and have been studied in the context of dark matter models [7, quantum gravity [13, string theory [3, and inflation [4].

For our purpose, we are going to solve this "classification problem" by using the branched covers and knot presentations to completely enumerate the smooth 4-manifolds. The fact that this works is simply due to the Montesinos-Piergallini theorem; any four-manifold can be presented by a 2-knot $\mathcal{K}_{2}$ and a covering space $p: M \rightarrow \mathbb{S}^{4}$, which itself is given by a particular representation of the fundamental group of the knot, $\rho_{j}: \pi_{1}\left(\mathbb{S}^{4} \backslash \mathcal{K}_{2}\right) \rightarrow \mathcal{S}_{n}$, indexed by the number of subgroups of $\pi_{1}\left(\mathbb{S}^{4} \backslash \mathcal{K}_{2}\right)$. So our geometries are collections $\left(\mathcal{K}_{2}^{(i)}, \rho_{j}, g\right) \equiv(i, j, g)$, and the partition function is now presented as

$$
Z=\sum_{(i, j, g)} e^{-\frac{1}{\hbar} S_{E H}[g]}
$$

and is formally complete. Of course, this is not everything we could ask for, since the classification problem is not solved; this is just a reparameterization of our ignorance. Rather than making this a sum over all elements of the complicated Dif $(M)$, we are summing over all of the well-defined 2-knots $K_{2}^{(i)}$, representations $\rho_{j}$, and metrics $g$. In addition, we are provided with a partial method to determine when a pair $\left(K_{2}, \rho, g\right),\left(K_{2}^{\prime}, \rho^{\prime}, g^{\prime}\right)$ are equivalent using standard techniques in knot theory.

Most importantly, by creating an explicit enumeration we can formally say that such a partition function is complete, whereas previously exotic smooth structures are "missed" because they are generally presented in rather abstract ways, almost always without a metric (for several examples, see Chapter 9 of [21). Using this approach, we are able to study particular classes of 4-manifolds without having to "worry" about exotic smooth structure. Of course, this is a little bit of a linguistic trick; the partition function (6) includes smooth manifolds $M^{\prime}$ which might normally be classified as "exotic" with respect to a homeomorphic manifold $M$. The point is that rather than being unsure about the existence of $M^{\prime}$ (because that would require checking all the possible diffeomorphisms of $M$ ), we know that if $M^{\prime}$ can be presented as a member of a particular class of $(i, j, g)$, we have included it. And of course, we know that $M^{\prime}$ can definitely be presented as some $(i, j, g)$.

Using the integral of the scalar curvature (3), our partition function is now

$$
Z=\sum_{(i, j, g)} \exp \left[\frac{\kappa}{\hbar}\left(n \int_{\mathbb{S}^{4}}(R-2 \Lambda)+4 \pi \sum_{i}\left(1-\alpha_{i}\right) A_{\Sigma_{i}}\right)\right] .
$$

We will proceed to an extended example of this shortly, but we would first like discuss the dimensional reduction implied by the expression. Since the curvature over the 4 -sphere is constant, $R=12 / r^{2}$, all the field dynamics are found in the second term, which is simply an area integral,

$$
\int d \zeta_{1} d \zeta_{2} \sqrt{d g^{(i)}}
$$

for a 2-dimensional metric $g^{(i)}$ on each of the singular surfaces $\Sigma_{i}$. Recall, these surfaces are normal to the 2-knots, and additionally the gravitational coupling constant for these surfaces is topological, $\gamma_{i}=\frac{4 \kappa}{\hbar} \pi\left(1-\alpha_{i}\right)$. They have no classical dynamics, but thinking of $g$ as a free quantum field we could construct generating functions and calculate transition probabilities between topological states represented as different branched covering spaces.

So rather then having to deal with general sets of smooth 4-manifolds (with both topological and geometrical data), we have reduced the problem to generic 2-manifolds with singular points and finite presentations on $\mathcal{S}_{n}$. This drastically simplifies the problem, since we are now out of the wild realm of 4-dimensional geometry and into the well-understood dimension 2. This dimensional reduction from four to two is commonly found when studying the quantum nature of the gravitational field (for a recent review, see 9]), and the above partition function is a new, independent example of this behavior.

For a more concrete realization of the dynamics implied by this action which emphasizes the dimensional reduction, we could rewrite the action on the surface by the standard Nambu-Goto string action,

$$
\sum_{i} \frac{T_{i}}{c} \int d \sigma d \tau \sqrt{\left(\dot{x} x^{\prime}\right)^{2}-(\dot{x})^{2}\left(x^{\prime}\right)^{2}}
$$

\footnotetext{
${ }^{1}$ Two particular manifestations of this: 1) there are simply-connected 4-manifolds with diffeomorphism groups that have infinitely many connected components [32], and 2) there are compact 4-manifolds whose diffeomorphisms group is not Jordan 11.
} 


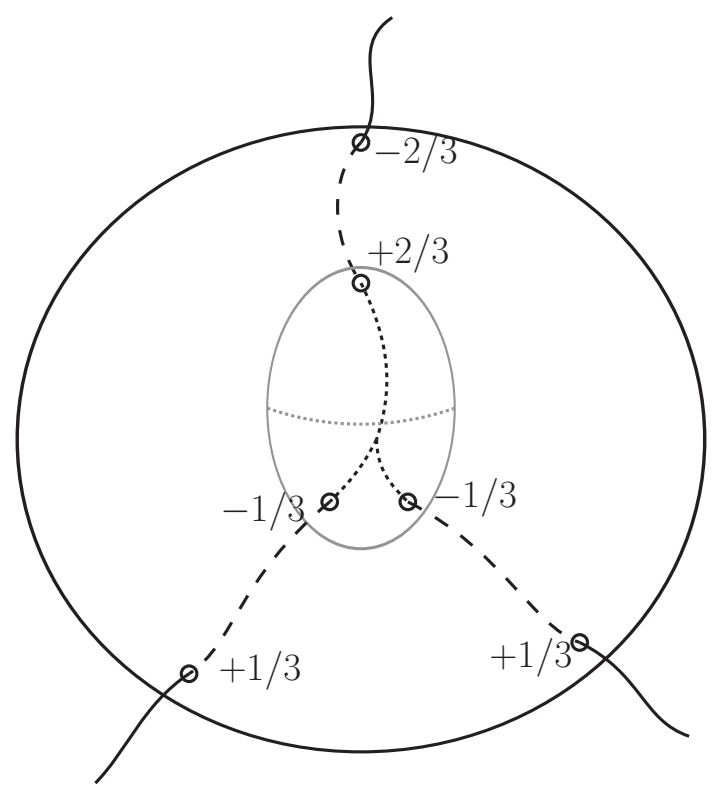

Figure 5: The splitting of a "topological" string.

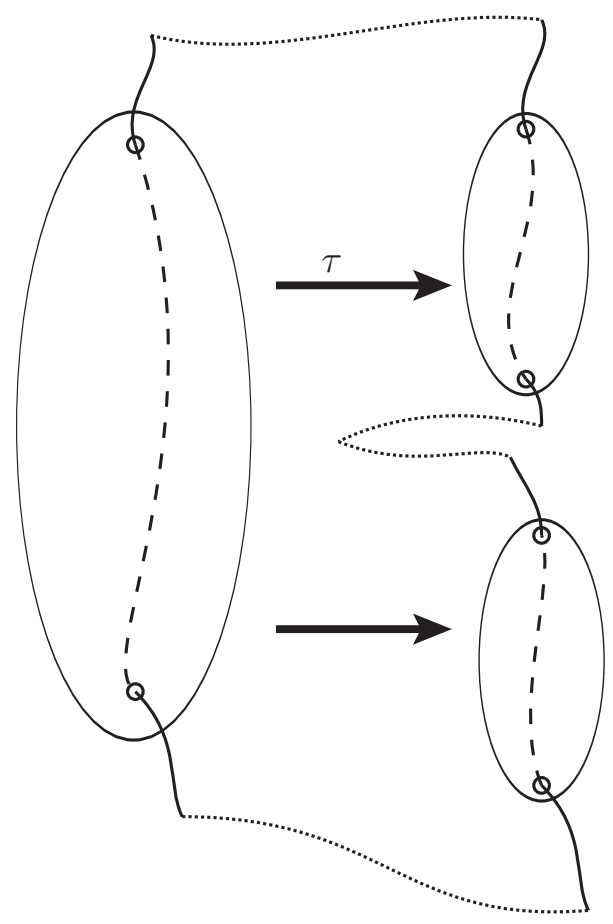

Figure 6: The splitting of a "geometric" string worldsheet, in which energy is conserved. Proper time travels to the right in this figure.

where $T_{i}$ is the "string tension" $T_{i}=4 \pi\left(1-\alpha_{i}\right) c$. Note that this does not need to be a physical string - it may simply represent a topological deficit, indistinguishable from a string except at very short distances. In this way, we can finally assign some physical characteristics to this model. The spatial section is topologically $\mathcal{K}_{2} \times \mathbb{S}^{1}($ or $\mathcal{K}_{2} \times I$ for an open string), and evolves along the proper time $\tau$ because of the form of the string action. The string worldsheet is dual to the 2 -knot, and we have corresponding $D$-branes, with $D=0,1$, or 2 . We can even describe the interactions of strings using our 2-knot cross-sections with non-locally flat points. This can happen in two distinct ways, depending on the physical restrictions one considers. The topological condition coming from the Gauss-Bonnet theorem (5) is required in all cases, and these could be considered "topological strings". However, physical strings must conserve energy as well, and these could be considered "geometric strings".

An example of a topological string can be seen in figure 5 In this case, we are representing a split string, with surfaces chosen to be tori with $\chi=0$. Of course, these types of strings are not usually considered in most approaches to either quantum or classical strings, because the strings are no longer submanifolds.

For geometric strings, we enforce energy conservation as well, which depends on the length of the string, $E_{i}=L_{i} T_{i}$. So when a string splits, the tension must change to conserve energy [39. In our model, this corresponds to the conical deficit changing during the interaction. For an example of this, see figure 6] If the initial string length is $L$, and the final string lengths are $f L$ and $(1-f) L$, we have a condition on the angle deficits coming from the condition of energy conservation,

$$
\left(1-\alpha_{i}\right) L=\left(1-\alpha_{1}\right) f L+\left(1-\alpha_{2}\right) L \rightarrow f=\frac{\alpha_{i}+\alpha_{2}}{\alpha_{1}+\alpha_{2}} .
$$

Since the angle deficit is not changing along the length of the string, each of these surfaces must be tori as well, with $\chi=0$. For example, picking $\alpha_{i}=2 / 3, f=1 / 3$, we have $\alpha_{1}=\alpha_{2}=2 / 3$ as one possibility.

\section{Example: The Preimage of a Trefoil in a 3-fold Branched Cover}

In this final section we will present a specific example of a set of model spacetimes parameterized with branched covering spaces and permutation groups discussed in this paper. We will arrive at a well-defined toy model which we will then use to calculate the thermodynamic entropy of the system. We will conclude this section with a few words emphasizing that while this is an abstract example, it stands in sharp contrast to what would be possible in 


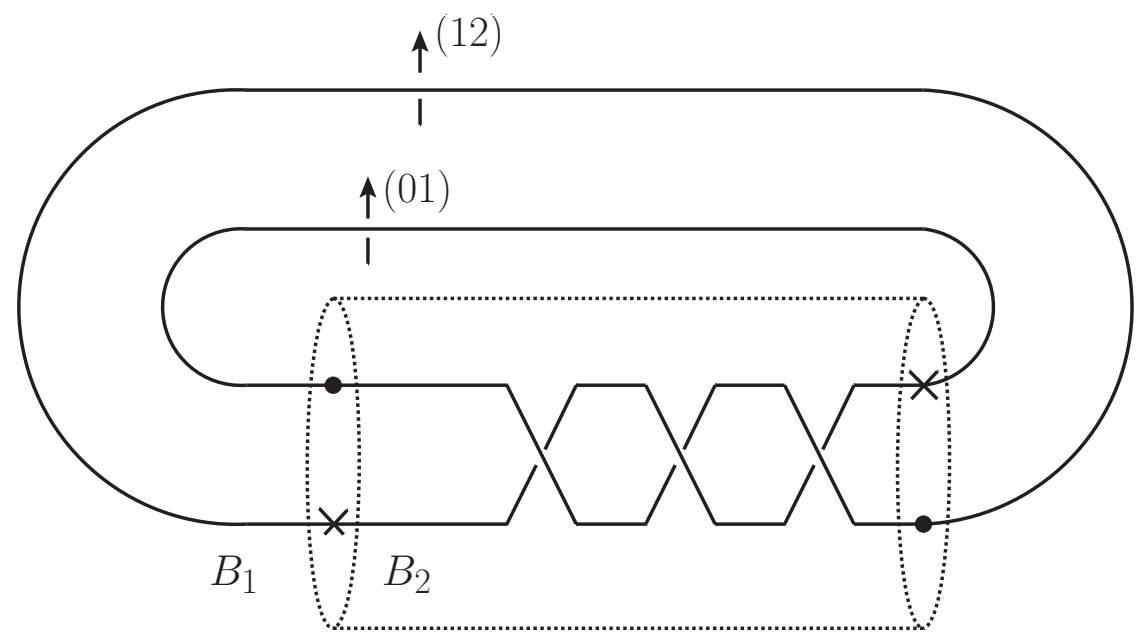

Figure 7: The trefoil in the base, with representation of the symmetric group

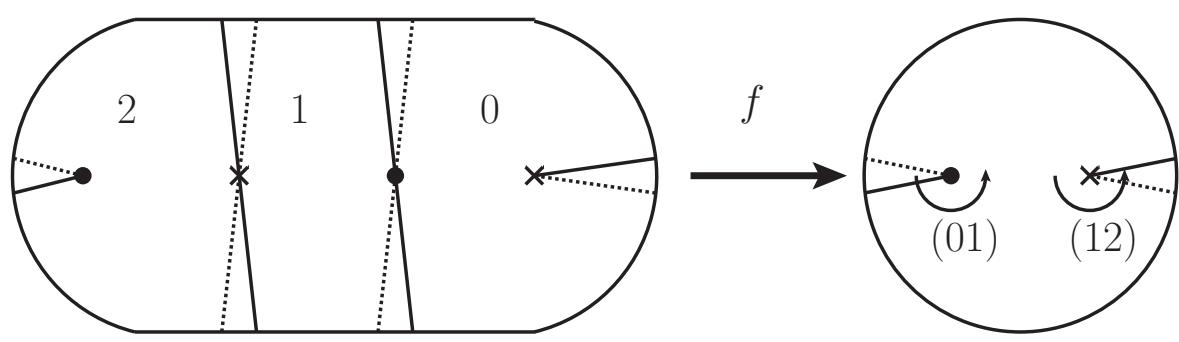

Figure 8: The branched cover over a slice through the ball $B_{2}$

the standard parameterization of the gravitational degrees of freedom.

The key piece of information we need is the preimages of the 1-knots $\left\{\mathcal{K}_{1}^{(t)}\right\}$ in the hyperplane cross-sections of a chosen 2-knot in the base, because those will represent the singularities found on the surface $p^{-1}(\Sigma)$. For concreteness, we will be fixing the order of the cover to be 3 . The inspiration for this example is the following classic example from Rolfsen 31: The 3-fold branched covering of $\mathbb{S}^{3}$ with ramification locus a (right-handed) trefoil is homeomorphic to $\mathbb{S}^{3}$. The ramification locus is the $(2,4)$ torus link.

\section{The Rolfsen Example}

First we will recall the original construction. Represent the trefoil $\mathcal{K}$ as a braid, shown in Figure 7 with two generators, $a$ and $b$. The covering space will be specified with the following representation of the symmetric group $\mathcal{S}_{3}$,

$$
\rho(a)=(01), \quad \rho(b)=(12) .
$$

The base $\mathbb{S}^{3}$ can be split into the union of 3-balls, $B_{1} \cup B_{2}$, and using the branched cover map $f: N^{3} \rightarrow \mathbb{S}^{3}$ we can write the cover as $N^{3}=f^{-1}\left(B_{1}\right) \cup f^{-1}\left(B_{2}\right)$. The splitting of the base is indicated in the figure - the braiding is located in $B_{2}$. The preimage of $\left.\mathcal{K}\right|_{B_{1}}$ is simply three copies of two arcs. We can determine the preimage of $\left.\mathcal{K}\right|_{B_{2}}$ by tracking what happens to the arcs as the braid is twisted in the base.

Representing $B_{2}=D^{2} \times I$, we know that the preimage of $B_{2}$ will be three copies of $D^{2} \times I$ sewed together in a particular way (Figure 8). The arrangement of the three copies of the open disk is apparent by considering how they are sewn together by the permutations. Viewing this as $B_{2} \times 0$, we want to know what happens to the preimages of each arc as we travel to $B_{2} \times 1$. The braid representation of the trefoil indicates that this is a $3 \pi$ twist. Based on these exchanges, we can draw a picture of the braid shown in figure9. This braiding is determined by simply tracking each arc in the cover; for example, the twist is clockwise, so the cross goes under the dot (passing from heavy line to dotted line) three times, and moves from the rightmost arc to the leftmost arc in the cover. The result is figure 9 , which is a $(2,4)$ torus link and can be checked using the braid generators, for instance. 


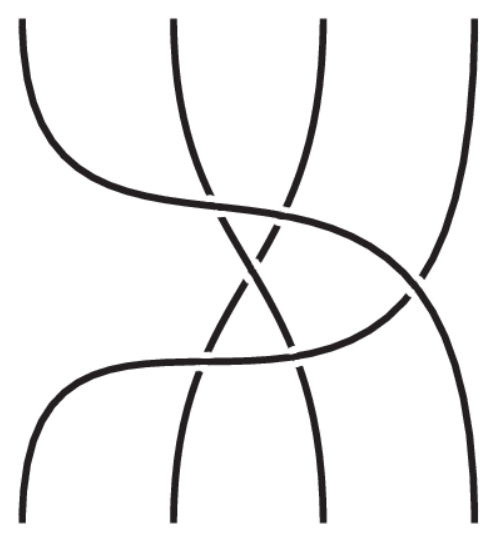

Figure 9: The braid in the covering space after a $3 \pi$ twist in the base.

This will be the starting point for our construction. We first want to determine the preimages of the trefoil under all possible 3-fold branched covers, then construct a set of model 4-manifolds branched over a particular 2-knot which has only trefoil or unknot singularities.

Before going any further, we should point out that a key feature of the previous example is that the branched cover had a trivial fundamental group, and therefore the class of any particular knot can be well-defined there. If the branched cover $p: N^{3} \rightarrow \mathbb{S}^{3}$ has $\pi_{1}(N) \neq 0$, we would not be able to unambiguously define what the knot class is. We would have to contend with questions like "how do arcs of the knot wrap around paths $\gamma \in \pi_{1}(N)$ ?". We could resolve this issue by simply restricting our attention to cases where $\pi_{1}(N)=0$, which can be checked by applying a version of the Reidemeister-Schreier due to Fox 19] (we will refer to this as "Fox's algorithm"). However, the manifolds $N^{3}$ will be embedded in the spacetime model $M^{4}$, so we actually need to be concerned with the topological structure of $M^{4}$ (because elements of $\pi_{1}(N)$ could be contractible in $M$ ). The actual topological conditions are that the preimages of the knot $\mathcal{K}$ in $M$ must be embedded in a topologically trivial sphere $\mathbb{S}^{3} \subset M$. However, this will all be subsumed by simply requiring that the knot group of the branched covers is trivial, which we will need to check for each case.

\section{Admissible Covers}

A particular representation $\rho: G \rightarrow \mathcal{S}_{3}$ is only admissible if the images of the group elements in $\mathcal{S}_{3}$ satisfy the relations of the knot presentation, in this case the trefoil:

$$
G=(a, b \mid a b a=b a b)
$$

First, we can count the number of possible covers because they will be given by pairs

$$
\left(\rho_{1}(a), \rho_{2}(b)\right)=\left(\rho_{1}, \rho_{2}\right) \in S_{3} \times S_{3},
$$

for 36 possible cases. We will break these into categories, and for each category we will check to see if the covers described by these representations are admissible.

- Cat 1: Transpositions which share a single sheet, as in Rolfsen's original example. These are of the form $(n p) \times(n q), q \neq p$, and there are 6 of them. These covers are all admissible:

$$
(n p)(n q)(n p)=(n q)(n p)(n q) \rightarrow(n)(p q)=(n)(p q)
$$

- Cat 2: One of the elements is the identity, $(1) \times \rho$. There are twelve of these of these, and in order to be admissible they must satisfy

$$
\text { (1) } \rho(1)=\rho(1) \rho \rightarrow \rho=\rho^{2}
$$

So only the case $\rho=(1)$ is admissible; call it Cat 2 a.

- Cat 3: Pairs of identical transpositions, $(p q) \times(p q)$ or $(p q m) \times(p q m)$. There are six of these, and they are all admissible. 


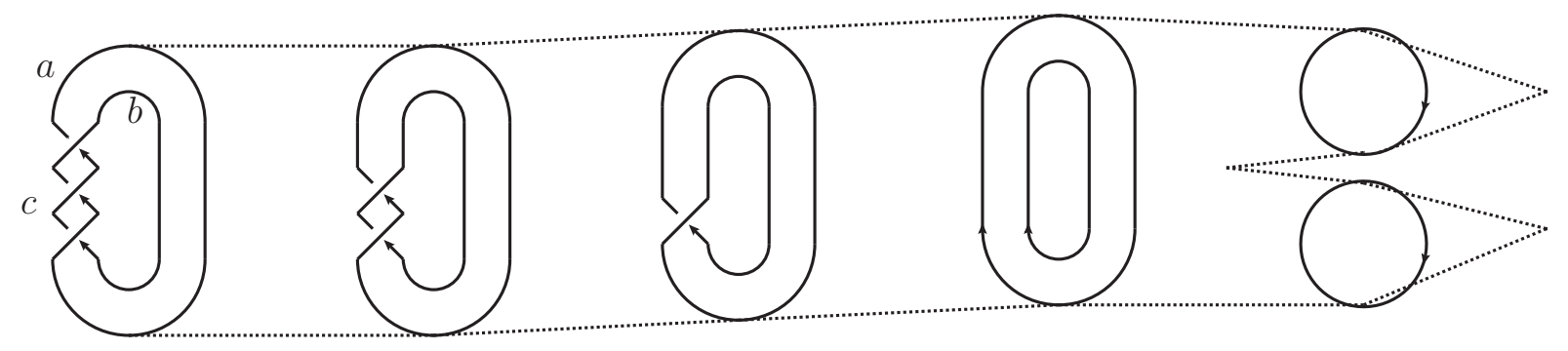

Figure 10: The particular branch set, represented with knot cross-sections.

- Cat 4: A 3-cycle and a 2-cycle. We have to work out each of these cases, but none of them are admissible.

$$
\begin{array}{lll}
(012)(01)(012)=(01)(2) \neq(01)(012)(10)=(021), & (012)(02)(012)=(02)(1) \neq(02)(012)(02)=(021) \\
(012)(12)(012)=(0)(12) \neq(12)(012)(12)=(021), & (021)(01)(021)=(01)(2) \neq(01)(021)(01)=(012) \\
(021)(02)(021)=(02)(1) \neq(02)(021)(02)=(012), & (021)(12)(021)=(0)(12) \neq(12)(021)(12)=(012)
\end{array}
$$

Taking into account the order of the permutations, this is 12 cases.

- Cat 5: Pairs of 3-cycles, of which there are two, neither of which are admissible. For example,

$$
(012)(021)(012)=(012) \neq(021)(012)(021)=(021)
$$

Checking $\pi_{1}\left(M \backslash p^{-1}\left(\mathcal{K}_{2}\right)\right)=0$

Now that we know which of these covers are admissible (Cat 1, Cat 2a, and Cat 3), we will check that the branched covering space corresponding to each does indeed have a trivial fundamental group. At this stage we have to specify a particular 2-knot, and we will pick the simplest possible example, with 1 singular point represented by a trefoil. The cross sections of this knot are shown in Figure (10). However, following the discussion in $\$ 5$ we must ensure that the Gauss-Bonnet theorem is satisfied. Fortunately, torus knots have the property that $(p, q) \simeq(q, p)$, so the angle deficits satisfy a kind of duality transformation, $\alpha \leftrightarrow 1 / \alpha$. So our trefoil knot will have $+2 / 3$, and the two knots at the bottom can be $(1,3)$ tori, with angular deficits $-1 / 3$ each (respecting the orientation), making this 2 -knot a torus. Note that although there appears to be a significant amount of freedom here, this is the only choice for a trefoil and two unknots consistent with Gauss-Bonnet. Naturally, the trefoil could split into an arbitrary number $m$ of unknots represented as torus knots $\left(1, n_{i}\right)$ as long as

$$
\frac{2}{3}+\sum_{i}\left( \pm \frac{1}{n_{i}}\right)=0
$$

counting orientations. A presentation for the fundamental group of the complement of this 2-knot in the base can be found by setting $a=b$ in the trefoil presentation, so $\pi_{1}\left(\mathbb{S}^{4} \backslash \mathcal{K}_{2}\right)=\mathbb{Z}$ (details on using the van Kampen theorem in this situation can be found in [19] and [6]).

The essence of Fox's algorithm ([19] and also see [14]) is a homomorphism $\phi$ between a presentation of the fundamental group of the knot complement in the base to the fundamental group of the $n$-fold cover times a free group:

$$
\phi: \pi_{1}\left(\mathbb{S}^{3} \backslash \mathcal{K}\right) \rightarrow H * F_{n-1} .
$$

For a word $u=x_{j_{1}}^{\epsilon_{1}} x_{j_{2}}^{\epsilon_{2}} \ldots$, we have a set of words associated to each sheet $0,1, \ldots, n-1$ of the cover,

$$
u_{\alpha}=\phi_{\alpha}(u)=x_{j_{1} \alpha_{1}}^{\epsilon_{1}} x_{j_{2} \alpha_{2}}^{\epsilon_{2}} \ldots
$$

where $\alpha_{k}=\rho_{k}(\alpha)$ and the permutation elements $\rho_{k}$ are given by the following rule:

$$
\epsilon_{k}=\left\{\begin{array}{cc}
+1, & \rho_{k}=\rho\left(x_{j_{k-1}-1}^{\epsilon_{k-1}}\right) \ldots \rho\left(x_{1}^{\epsilon_{1}}\right) \\
-1, & \rho_{k}=\rho\left(x_{j_{k}}^{\epsilon_{k}}\right) \ldots \rho\left(x_{1}^{\epsilon_{1}}\right)
\end{array}\right.
$$

Here the $\rho(x)$ are the topological labels on each edge. Acting on each generator and relation in $\pi_{1}\left(\mathbb{S}^{3} \backslash \mathcal{K}\right)$, the fundamental group of the cover can be recovered from $H * F_{n-1}$ by 
- Removing the free group by setting elements of a chosen Schreier tree to zero, and

- Ensuring curves that represent the preimage of the branch locus are trivial. For such an element $v$ of the branch locus represented by $\rho(v)=\left(\beta_{1} \ldots \beta_{\lambda}\right)(\ldots) \ldots$ (in cycle notation) we adjoin the relations

$$
\phi_{\beta_{1}}(v) \ldots \phi_{\beta_{\lambda}}(v)=1, \ldots
$$

To illustrate this calculation, let us expand the original Rolfsen example and determine the knot group in the cover. In other words we will determine what the fundamental group is over a 3 -fold cover of the trefoil given by the representation $\rho(a)=(01)(2)$ and $\rho(b)=(0)(12)$. Under the homomorphism $\phi$, the presentation becomes

$$
\left(a_{0}, a_{1}, a_{2}, b_{0}, b_{1}, b_{2} \mid a_{0} b_{1} a_{2}=b_{0} a_{0} b_{1}, a_{1} b_{0} a_{1}=b_{1} a_{2} b_{1}, a_{2} b_{1} a_{0}=b_{2} a_{1} b_{2}\right) .
$$

A Schreier tree is given by

which under the homomorphism gives

$$
w_{0}=1, \quad w_{1}=a, \quad w_{2}=a^{2},
$$

$$
w_{00}=1, \quad w_{10}=a_{0}, \quad w_{20}=a_{0} a_{1} .
$$

So to remove the free group we set $a_{0}=1$ and $a_{1}=1$, which makes the presentation

$$
\left(a_{2}, b_{0}, b_{1}, b_{2} \mid b_{1} a_{2}=b_{0} b_{1}, b_{0}=b_{1} a_{2} b_{1}, a_{2} b_{1}=b_{2} b_{2}\right) .
$$

To trivialize the branch locus, we set

$$
a_{0} a_{1}=1, \quad a_{2}=1, \quad b_{0}=1, \quad b_{1} b_{2}=1 .
$$

The presentation is then

$$
\left(b_{1}, b_{2} \mid 1=b_{1}^{2}, b_{1}=b_{2}^{2}, b_{1} b_{2}=1\right) .
$$

The only way this set of relations is consistent is if $b_{1}=b_{2}=1$, and the fundamental group of the complement in the cover is trivial.

Now we need to extend this to our example, with the trefoil being a cross section of the knot in figure 10 . In the base, the knot group will be the same as the trefoil, $(a, b \mid a b a=b a b)$, but with the additional relation $a=b$. This eliminates Cat 1 from our considerations, because that requires $\rho(a) \neq \rho(b)$.

For Cat $2 \mathrm{a}, \rho(a)=\rho(b)=(0)(1)(2)$, and the result is a disconnected space with topology $\mathbb{S}^{4} \cup \mathbb{S}^{4} \cup \mathbb{S}^{4}$. So this space is not simply connected, but since it's completely disconnected, there will be a copy of $p^{-1}\left(\mathcal{K}_{2}\right)$ in each component, and the knot type is well-defined in each of them.

In Cat 3 , we have 2 prototype cases to cover, $(01) \times(01)$ and $(012) \times(012)$. In the first type, one sheet is always left untouched, so it will also be disconnected, with topology $N^{\prime} \cup \mathbb{S}^{4}$. Under the homomorphism, the image of the presentation will be

$$
\left(a_{0}, a_{1}, a_{2}, b_{0}, b_{1}, b_{2} \mid a_{0} b_{1} a_{0}=b_{0} a_{1} b_{0}, a_{1} b_{0} a_{1}=b_{1} a_{0} b_{1}, a_{2} b_{2} a_{2}=b_{2} a_{2} b_{2}, a_{0}=b_{0}, a_{1}=b_{1}, a_{2}=b_{2}\right) .
$$

The third relation is the disconnected piece, but with $a_{2}=b_{2}$ we have just another copy of the base. Using the same tree as above and eliminating the $b$ s, we are left with a single generator $a_{2}$ and no relations. Trivializing the branch locus tells us $a_{2}=1$, so that case is simply connected (although this actually means $\mathbb{S}^{4} \cup \mathbb{S}^{4}$ ).

Finally, we have the 3-cycle prototype in Cat 3. The homomorphism gives us

$$
\left(a_{0}, a_{1}, a_{2}, b_{0}, b_{1}, b_{2} \mid a_{0} b_{1} a_{2}=b_{0} a_{1} b_{2}, a_{1} b_{2} a_{0}=b_{1} a_{2} b_{0}, a_{2} b_{0} a_{1}=b_{2} a_{0} b_{1}, a_{0}=b_{0}, a_{1}=b_{1}, a_{2}=b_{2}\right),
$$

which is connected. Using the same tree and removing the $b \mathrm{~s}$, we are again left with a single generator, $a_{2}$, which becomes trivial when taking the branch locus into account:

$$
a_{0} a_{1} a_{2}=1
$$

Now that we have determined that the knot complement group in each cover is trivial, we need to determine the preimage of the trefoil and each unknot.

\section{Preimages of (1,3)-Torus Knots}

The preimages of the $(1,3)$ torus knots (equivalent to unknots) are straightforward to determine. Since there is no "twist", like that found in the braid representation of $(2,3)$, we simply get a copy of a $(1,3)$ in each sheet of the cover, the location of which is determined by the permutation label. For instance, for $\rho(a)=\rho(b)=(0)(12)$, there is one copy for sheet 0 and one copy for the sheet linked by the permutation (12). 

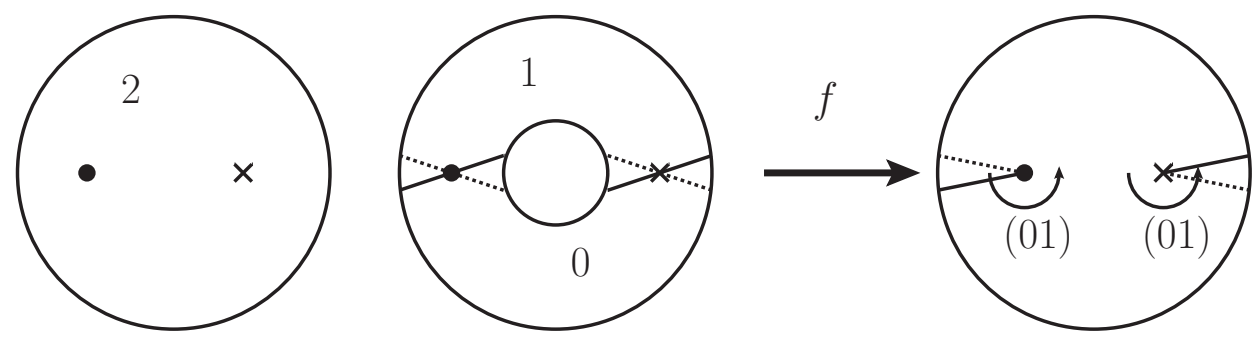

Figure 11: An example of a cover from Cat 3, for two equal transpositions.

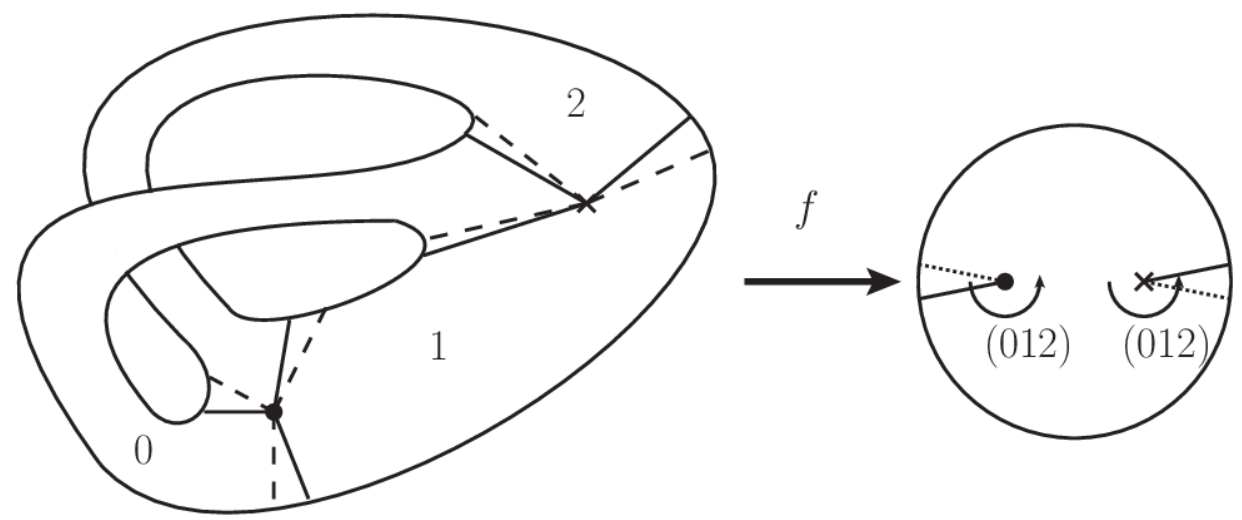

Figure 12: An example of a cover from Cat 3, for two equal 3-cycles.

\section{Preimages of $(2,3)$-Torus Knots}

For the preimages of the trefoil, we will need to follow the technique outlined in Rolfsen's original example. Starting with Cat 2, we have to consider each sheet separately. Since there are three disjoint copies of the base, the preimage will be 3 copies of the trefoil.

For Cat 3, we'll do the transposition case first, shown in figure 11 Since we know this cover is embedded in a topologically trivial manifold $M^{4}$, the hole in the annulus will go away. Under the clockwise $3 \pi$ twist in the base, both pairs of arcs simply twist around each other three times, resulting in two copies of the $(2,3)$ torus (see figure 7 ).

For pairs of identical 3-cycles, the slice through the cover is shown in Figure 12 There is only a single preimage of each arc, so this is a braid on a single generator. Under the $3 \pi$-twist, the cross travels over the dot 3 times and this is a single copy of the $(2,3)$ torus.

\begin{tabular}{c|c|c|c} 
Permutation Class & Number & Torus Type of the Preimage of $(2,3)$ & Torus Type of the Preimage of $(1,3)$ \\
\hline$(1) \times(1)$ & 1 & $(2,3) \cup(2,3) \cup(2,3)$ & $(1,3) \cup(1,3) \cup(1,3)$ \\
$(n p) \times(n p)$ & 3 & $(2,3) \cup(2,3)$ & $(1,3) \cup(1,3)$ \\
$(n p q) \times(n p q)$ & 3 & $(2,3)$ & $(1,3)$
\end{tabular}

So finally, we have a complete list of states for this particular model. In the end, we just get generalizations of the 2-knot shown in figure 10. For instance, for the covers represented by the permutation class $(p q) \times(p q)$, the 2-knot is a locally flat surface with two $(2,3)$-torus singularities and four $(1,3)$-torus singularities. Notice we don't actually know how these cross sections are precisely arranged without a closer inspection of the crossing regions in the base, but we don't need that to determine the partition function (7).

The partition function can be split into the contribution from the base and the contribution from the cover,

$$
Z=\left(\exp \left[\frac{3 \kappa}{\hbar} \int R d V\right]\right)\left(\sum_{(i, j, g)} \exp \left[4 \pi \frac{\kappa}{\hbar} \sum_{k}\left(1-\alpha_{k}\right) \int \delta(\Sigma(i, j)) d V\right]\right)=Z_{0} \bar{Z}
$$

where we have set $\Lambda=0$. Of course, this means the action is negative-definite and the partition function formally divergent. This could be avoided by using an Einstein metric on the base sphere, but that modification is easy to make from our more simplified and direct approach. 
Considering the radius of the base sphere $R$ as a constant, the first piece can be determined explicitly 2,

$$
Z_{0}=\exp \left(\frac{36 \pi^{2} \kappa}{\hbar} R^{2}\right)
$$

To work out the second piece, we will first assume that the covering maps are isometric; that is, the metric on $p^{-1}(\Sigma)$ in the covers matches the metric in the base $\Sigma$. In that case, $A_{\Sigma}=\int \delta(\Sigma) d V$ in the sum. Now calling the number of singularities on the $i$ th preimage of each $2-\operatorname{knot} n_{i}$, we can write the sum over the angle deficits as

$$
\sum_{k=1}^{n_{i}}\left(1-\alpha_{k}\right)=n_{i}-\chi\left(\Sigma_{i}\right)=n_{i}
$$

since in our particular case we have $\chi\left(\Sigma_{i}\right)=0$. This piece now looks like

$$
\bar{Z}=\sum_{i} \exp \left(\frac{4 \pi \kappa n_{i}}{\hbar} A_{\Sigma}\right)
$$

It is pretty clear that the area $A_{\Sigma}$ is playing the role of the classical (inverse) temperature in this system. With that in mind, we can cast this partition function in the usual form by defining a physical constant $\gamma$ with units of energy per unit area to play the role of the Boltzmann constant, and define

$$
\beta=\gamma A_{\Sigma}, \quad E_{i}=\frac{4 \pi \kappa}{\hbar \gamma} n_{i}
$$

so our partition function looks like

$$
Z=\sum_{i} g_{i} \exp \left(E_{i} \beta\right)
$$

with $g_{i}$ denoting the degeneracy of the "energy level" $E_{i}$, where the energy now just depends on the number of singularities in the preimage of the knot. Using standard techniques from statistical mechanics, we can calculate the free energy as $F=-\frac{1}{\beta} \ln (Z)$, and the entropy is

$$
S=\frac{36 \pi^{2} \kappa}{\hbar} R^{2}+\ln \left(\sum g_{i} \exp \left(E_{i} \beta\right)\right)+\frac{\sum E_{i} g_{i} \beta \exp \left(E_{i} \beta\right)}{\sum \exp \left(E_{i} \beta\right)}=S_{0}+S^{\prime}
$$

The first term in this expression is dependent on the size of the base sphere, while the second two terms only depend on the 2-knots in the branched cover. We can study the scaling properties of this system by varying the ratio of the physical sizes, $\delta=R^{2} / A_{\Sigma}$, and setting the units to be $\kappa /(\hbar \gamma)=1$. When $\delta \approx 1$, the relative contributions to the entropy are approximately equal when $\beta \approx 0.01$. As $\delta \rightarrow 0$, the temperature at which this transition occurs decreases dramatically, see figure 13 .

We would like to contrast this calculation with an equivalent one using more conventional approaches to semiclassical gravity. Say we want to start off with a very physically reasonable model space (say, a 4-sphere), and consider the set of states which are "close" to it - topologically the same but have different geometries. Even this most basic classification is impossible; since the smooth Poincaré conjecture is unsolved in dimension 4, we have no idea how many smooth 4-spheres there are which are gauge-inequivalent to the standard one, and we have no idea how they might be presented. Compared to our example, which lacks some physical meaning but is very well-presented as a statistical model, the advantage is clear. It is this stark contrast which is the primary motivation for this particular presentation of spacetime.

\section{Summary}

A major feature of our modern understanding of the universe is the importance of the spacetime model. Fundamentally geometric objects, the necessary complexity of the spacetime models used in general relativity presents a major challenge when trying to study them either in detail or generally. In this work, we reviewed a proposal to reparameterize the spacetime model into a branched covering space over a model sphere, and applied the construction to the case in which the branching set was a 2 -knot.

There are several advantages to this approach. The first is the ability to study general classes of smooth 4manifolds as model spacetimes. Although some of the physical characteristics of the spacetimes have been lost, the lost information is replaced with an algorithmic method to construct all such spacetimes. In this way calculations

\footnotetext{
${ }^{2}$ One possibly interesting generalization of this would be to consider covering maps in which the radius $R$ of the base space is allowed to vary freely. This could correspond to an expanding universe with topology change, such as [4]
} 


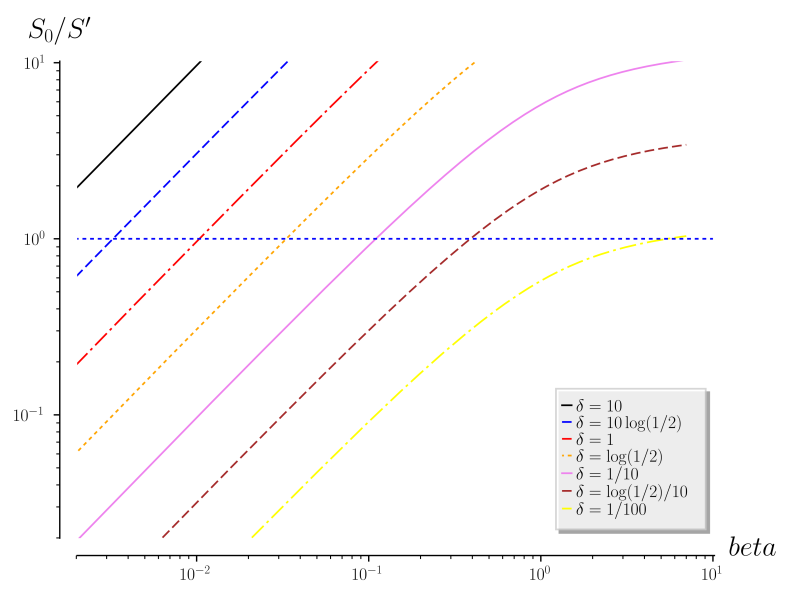

Figure 13: A plot of the temperature parameter $\beta$ against the ratio of the entropy contributions from the base sphere $S_{0}$ and the spacetime model $S^{\prime}$.

requiring a sum-over-states can be well-posed, and the presence of exotic smooth structure is incorporated in a natural way. In addition, and in stark contrast to the usual approaches, the topology of the spacetime model can be explicitly tracked and studied.

When the branched covers are presented over 2-knots as they have been here, a number of abstract features of these models become more concrete. In particular, the singularities in the covering space are made explicit, and are directly related to the knot and the covering presentation in the base. This is easiest to see when the knots are chosen to be tori. It becomes possible to choose a set of kinematic states for the gravitational field and perform semiclassical calculations without having to directly solve the classification problem, which has stumped mathematicians for decades.

There are a number of directions in which this exploration could be continued. For the specific example presented here, it would be interesting to take Rolfsen's example to it's natural conclusion, and work out a generic way to determine the preimage of any knot in a branched cover. As far as we know, this is a completely open question and a possible source of rich mathematical knowledge. If this was done, it would be possible to study a very large class of model spacetimes in a statistical manner, such as what we presented in $\$ 7$ Even in the more restricted case that we've presented, other questions could be answered via the statistical physics/QFT interpretation. For instance, one could explore the tunneling probability between topologically inequivalent states. Or, by adding a source term $J_{a b}$ to the generating function, e.g.

$$
Z=\exp \left(S_{0}+J^{a b} h_{a b}\right),
$$

one could perform loop calculations, study ghost contributions, etc. And since the topology is being tracked by the construction, we would finally have a model for spacetime in which the topology is determined dynamically, rather then a priori. Perhaps this would provide some clue as to what happens to spacetime topology when one passes to the quantum realm, but more generally it would expand the characteristic background independence of the gravitational field to include both the geometry and the topology.

Before we conclude this paper, we would also like to point out a higher-order theme to this work, which is a further exploration of the deep connection between physics and mathematics. The primary inspiration for this has been the discovery and exploration of exotic smooth structures in dimension 4, which utilized methods from both differential topology and geometry but also gauge theory and analysis. In this work, we are suggesting a new connection between the rich and well-studied mathematical field of knot theory and general relativity. Specifically, the appearance and importance of the non-locally flat points in our approach demonstrates that without a deep understanding of 1- and 2-knots on one hand and action integrals on the other, this exploration would have been impossible. More generally, we would like to propose the following paradigm; if spacetime is a fundamentally geometric object, all alternative representations of the geometry must be viewed as equivalent and should be explored and evaluated for use in physical calculations.

Thus, in the end, this study is about taking the geometric nature of the gravitational field seriously. Since the manifold structure which allows us to perform calculations and verify our cosmological models necessarily depends on all aspects of this geometry - points, sets, topology, and smooth structure - alternative presentations which also include this data represent fertile grounds for productive research. For the branched covering reparameterization, 
we trade off physical intuition in favor of well-posed questions, but are left with interesting toy models to examine for clues into the deeper mysteries of the four-dimensional world. It is our hope that this project will inspire other members of the community to explore this and other geometrically-motivated reparameterization of the gravitational field, to not only gain a more complete understanding of our spacetime models, but to also foster new connections between theoretical physics, observations, and mathematics.

\section{Acknowledgments}

This work was partially supported by a 2017 Faculty Development grant from Merrimack College. The author would also like to thank the organizers of the First Hermann Minkowski Meeting on the Foundations of Spacetime Physics for the opportunity to present this work to those in attendance.

\section{References}

[1] J. W. Alexander. Note on Riemann spaces. Bull. Amer. Math. Soc., 26(8):370-372, 1920.

[2] T. Asselmeyer-Maluga and C. H. Brans. Exotic smoothness and physics. World Scientific Publishing Co. Pte. Ltd., Hackensack, NJ, 2007.

[3] T. Asselmeyer-Maluga and J. Krol. Exotic smooth R^4, geometry of string backgrounds and quantum D-branes. ArXiv e-prints, June 2010.

[4] T. Asselmeyer-Maluga and J. Król. How to obtain a cosmological constant from small exotic $\mathrm{R}^{4}$. Physics of the Dark Universe, 19:66-77, Mar. 2018.

[5] B. Bode and M. R. Dennis. Constructing a polynomial whose nodal set is any prescribed knot or link. ArXiv e-prints, Dec. 2016.

[6] J. Boersema and E. Whitaker. Knots in four dimensions and the fundamental group. Rose-Hulman Undergraduate Mathematics Journal, 4(2), 2003.

[7] C. H. Brans. Localized exotic smoothness. Classical Quant. Grav., 11(7):1785-1792, 1994.

[8] G. Burde and H. Zieschang. Knots, volume 5 of de Gruyter Studies in Mathematics. Walter de Gruyter \& Co., Berlin, 1985.

[9] S. Carlip. Dimension and dimensional reduction in quantum gravity. Classical and Quantum Gravity, 34(19):193001, Oct. 2017.

[10] R. H. Crowell and R. H. Fox. Introduction to knot theory. Based upon lectures given at Haverford College under the Philips Lecture Program. Ginn and Co., Boston, Mass., 1963.

[11] B. Csikós, L. Pyber, and E. Szabó. Diffeomorphism Groups of Compact 4-manifolds are not always Jordan. ArXiv e-prints, Nov. 2014.

[12] D. Denicola, M. Marcolli, and A. Zainy al-Yasry. Spin foams and noncommutative geometry. Classical Quant. Grav., 27(20):205025, Oct. 2010.

[13] C. L. Duston. Exotic smoothness in four dimensions and Euclidean quantum gravity. Int. J. Geom. Methods Mod. Phys., 8(3):459-484, 2011.

[14] C. L. Duston. Topspin Networks in Loop Quantum Gravity. Classical Quant. Grav., 29:205015, 2012.

[15] C. L. Duston. Exotic smoothness, branched covering spaces, and quantum gravity. PhD thesis, The Florida State University, 2013.

[16] C. L. Duston. Semiclassical partition functions for gravity with cosmic strings. Classical and Quantum Gravity, 30(16):165009, Aug. 2013.

[17] C. L. Duston. The Fundamental Group of a Spatial Section Represented by a Topspin Network. ArXiv e-prints, Aug. 2013.

[18] C. L. Duston. Using cosmic strings to relate local geometry to spatial topology. International Journal of Modern Physics D, 26:1750033-583, 2017.

[19] R. H. Fox. A quick trip through knot theory. In Topology of 3-Manifolds and related topics, pages 120-167. Prentice-Hall, 1962.

[20] D. V. Fursaev and S. N. Solodukhin. Description of the Riemannian geometry in the presence of conical defects. Phys. Rev. D (3), 52(4):2133-2143, 1995. 
[21] R. E. Gompf and A. I. Stipsicz. 4-manifolds and Kirby calculus, volume 20 of Graduate Studies in Mathematics. American Mathematical Society, Providence, RI, 1999.

[22] H. W. Hamber. Quantum Gravitation: The Feynman Path Integral Approach. Springer-Verlag, Berlin, 2009.

[23] S. W. Hawking. The path-integral approach to quantum gravity. In S. W. Hawking \& W. Israel, editor, General Relativity: An Einstein centenary survey, pages 746-789, 1979.

[24] H. M. Hilden. Three-fold branched coverings of $S^{3}$. Amer. J. Math., 98(4):989-997, 1976.

[25] M. Iori and R. Piergallini. 4-manifolds as covers of the 4-sphere branched over non-singular surfaces. Geom. Topol., 6:393-401, 2002.

[26] M. Marcolli and A. Zainy al-Yasry. Coverings, correspondences, and noncommutative geometry. Journal of Geometry and Physics, 58:1639-1661, Dec. 2008.

[27] J. Milnor. On the 3-dimensional Brieskorn manifolds $M(p, q, r)$. Ann. of Math. Studies, No. 84, pages 175-225, 1975.

[28] J. M. Montesinos. Three-manifolds as 3-fold branched covers of $S^{3}$. Quart. J. Math. Oxford Ser. (2), 27(105):8594, 1976.

[29] R. Piergallini. Four-manifolds as 4-fold branched covers of $S^{4}$. Topology, 34(3):497-508, 1995.

[30] V. V. Prasolov and A. B. Sossinsky. Knots, links, braids and 3-manifolds, volume 154 of Translations of Mathematical Monographs. American Mathematical Society, Providence, RI, 1997.

[31] D. Rolfsen. Knots and links. Publish or Perish, Inc., Berkeley, Calif., 1976. Mathematics Lecture Series, No. 7.

[32] D. Ruberman. A polynomial invariant of diffeomorphisms of 4-manifolds. In Proceedings of the Kirbyfest (Berkeley, CA, 1998), volume 2 of Geom. Topol. Monogr., pages 473-488. Geom. Topol. Publ., Coventry, 1999.

[33] M. Salvetti. On the number of nonequivalent differentiable structures on 4-manifolds. Manuscripta Math., 63(2):157-171, 1989.

[34] A. Scorpan. The wild world of 4-manifolds. American Mathematical Society, Providence, RI, 2005.

[35] J. Seade. On the topology of isolated singularities in analytic spaces, volume 241 of Progress in Mathematics. Birkhäuser Verlag, Basel, 2006.

[36] T. Thiemann. Modern canonical quantum general relativity. Cambridge Monographs on Mathematical Physics. Cambridge University Press, Cambridge, 2007.

[37] A. Vilenkin and E. P. S. Shellard. Cosmic strings and other topological defects. Cambridge Monographs on Mathematical Physics. Cambridge University Press, Cambridge, 1994.

[38] A. Zorich. Flat surfaces. In Frontiers in number theory, physics, and geometry. I, pages 437-583. Springer, Berlin, 2006.

[39] B. Zwiebach. A first course in string theory. Cambridge University Press, Cambridge, 2004. 